\title{
A New ANFIS Model based on Multi-Input Hamacher T-norm and Subtract Clustering
}

\author{
Feng-Yi Zhang and Zhi-Gao Liao*
}

Department of Management, Guangxi University of Science and Technology, Liuzhou, Guangxi, China 545000

\begin{abstract}
This paper proposed a novel adaptive neuro-fuzzy inference system (ANFIS), which combines subtract clustering, employs adaptive Hamacher T-norm and improves the prediction ability of ANFIS. The expression of multi-input Hamacher T-norm and its relative feather has been originally given, which supports the operation of the proposed system. Empirical study has testified that the proposed model overweighs early work in the aspect of benchmark Box-Jenkins dataset and may provide a practical way to measure the importance of each rule.
\end{abstract}

Keywords: ANFIS, hamacher T-norm, subtract clustering, T-norm.

\section{INTRODUCTION}

Takagi, Sugeno and Kang have established what is called the Takagi-Sugeno-Kang (TSK) method [5-7]. This neuralnetwork-based fuzzy reasoning scheme is capable of learning the membership function of the "IF" part and determining the amount of control in the "THEN" part of the inference rules. What's more, it is nicely suited to mathematical analysis and usually works well with optimization and adaptive techniques. Subsequently, many improved algorithms and extensions were developed for the TSK model. In particular, the adaptive neuro-fuzzy inference system (ANFIS) is an important approach to implement the TSK fuzzy system, which has been put forward by Jang in 1993 [3]. However among all early work, only Iliadis et al. have replaced the algebraic product T-norm with other fuzzy T-norms to handle intersection operation [1]. But they have not explained the reason why they select the one. Besides, in the case of input dimension increasing, the number of rules will increase with the input dimension exponentially, which inevitably leads the conventional ANFIS structure dimension to disaster. In order to improve the online access speed of ANFIS T$\mathrm{S}$ rules for complex system, various clustering algorithms have been used to construct a new multidimensional structure of ANFIS, which combined mechanism of T-S fuzzy inference and clustering algorithm from the perspective of knowledge discover.In this paper, we select Hamacher Tnorm to tackle the intersection operation for two reasons:

1. Algebraic product T-norm is used widely in ANFIS, and when $\lambda$ equals to 1 , the Hamacher T-norm is actually an algebraic product T-norm, which means it is not conflicting with the regular ANFIS.

2. Hamacher product T-norm, a clustering of fuzzy product T-norms, differs depending on $\lambda$. So, to select the most suitable fuzzy T-norm by changing the numberical parameter $\lambda$ is advisable. Subtract clustering, which could obtain the amount and value of clustering center, was used to determine the If part of each rule, for its wide application in ANFIS center determination.

The rest of the paper is organized as follows. Section 2 provides some necessary background information, and the proposed system and its essential interference are discussed in Section 3. Section 4 presents the simulation results for benchmark Box-Jenkins dataset. Finally, the summary of this paper is given in Section 5.

\section{BACKGROUND}

In this section, the basic theory of ANFIS model and normalization method which has been used in this experiment will be introduced.

\subsection{Adaptive Network Based Fuzzy Inference System (ANFIS)}

Both artificial neural network and fuzzy logic are used in ANFIS architecture. ANFIS consists of if-then rules and couples of input-output. For ANFIS training, learning algorithms of neural network are also used. To simplify the explanations, the fuzzy inference system under consideration is assumed to have two inputs (x and y) and one output (f). For a regular ANFIS model, a typical rule set with basic fuzzy ifthen rules can be expressed as if $\mathrm{x}$ is $A_{1}$ and $\mathrm{y}$ is $B_{1}$, then

$f_{1}=p_{1} x+q_{1} y+r_{1}$

where $p$ is linear output parameters. The ANFIS architecture with two inputs and one output are as shown in Fig. (1).

This architecture is formed by five layers and nine if-then rules:

Layer-1: Every node $\mathrm{i}$ in this layer is a square node with a node function. 


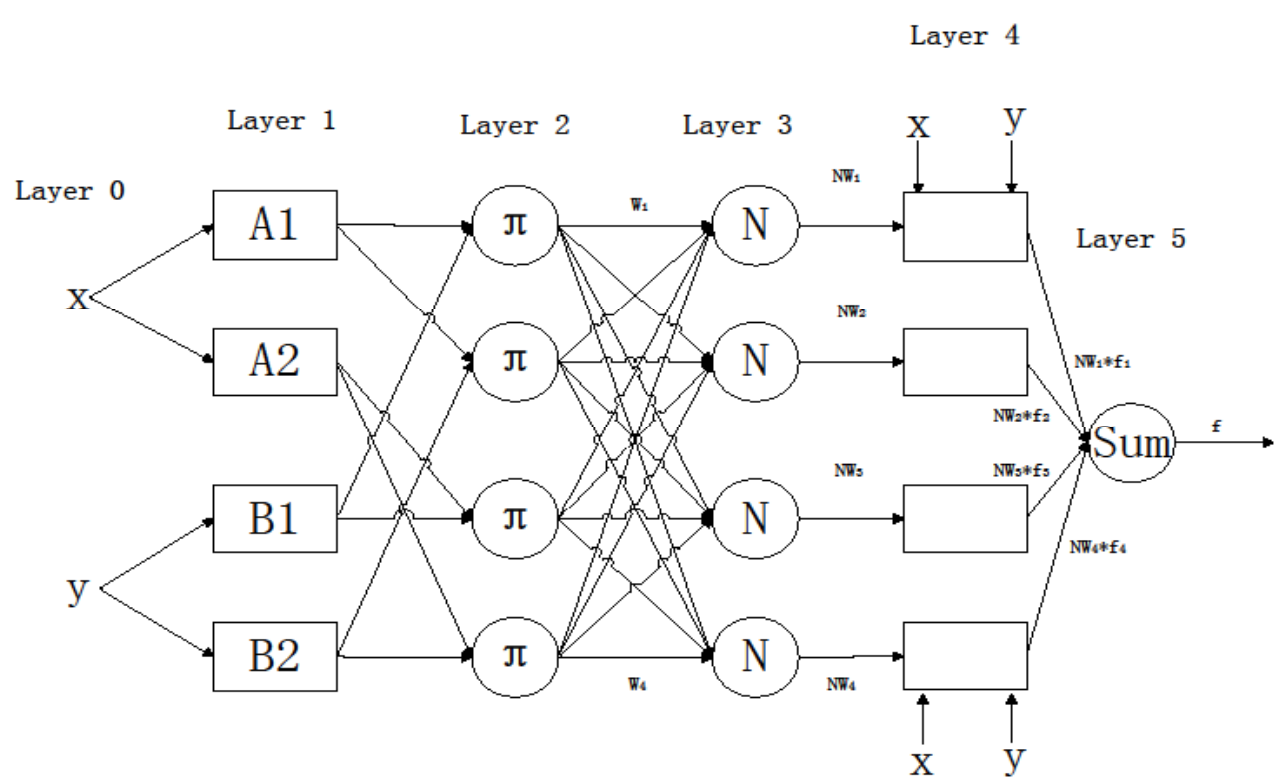

Fig. (1). The structure of regular ANFIS.

$O_{1, i}=\mu_{A_{i}}(x), O_{1,3+j}=\mu_{B_{j}}(y) \quad i, j=1,2$

where $\mathrm{x}$ and y are inputs to node $\mathrm{i}$, and $A_{i}$ and $B_{i}$ are linguistic labels for inputs. In other words, $\mathrm{O}_{1, i}$ is the membership function of $A_{i}$ and $B_{i}$. Usually $\mu_{A i}(\mathrm{x})$ and $\mu_{B i}(\mathrm{y})$ are chosen to be bell-shaped with maximum equalling to 1 and minimum equalling to 0 , such as

$\mu_{A_{i}}(x)=\exp \left(-\left(\left(x-a_{i}\right) /\left(c_{i}\right)\right)^{2}\right)$

where $\mathrm{a}_{i}, \mathrm{c}_{i}$ is the parameter set. These parameters in this layer are referred to as premise parameters.

Layer-2: Every node in this layer multiplies the incoming signals and sends the product out. For instance,

$O_{2,2(i-1)+j}=\mu_{A_{i}}(x) * \mu_{B_{j}}(y), i, j=1,2$

Each node output represents the firing strength of a rule.

Layer-3: Every node in this layer calculates the ratio of the rule's firing strength to the sum of all rule??s firing strengths:

$O_{3, i}=\widetilde{w}_{i}=w_{i} /\left(w_{1}+w_{2}+\cdots+w_{9}\right), i=1,2, \cdots, 4$

Layer-4: Every node $\mathrm{i}$ in this layer is a square node with a node function

$O_{4, i}=\widetilde{w}_{i} * f_{i}=\tilde{w}_{i}\left(p_{i, 1} x_{1}+p_{i, 2} x_{2}\right), i=1,2, \cdots, 4$

where $w_{i}$ is the output of layer 3 and $p_{i, 1}, p_{i, 2}, p_{i, 3}$ is the parameter set. Parameters in this layer will be referred to as consequent parameters.

Layer-5: The single node in this layer computes the overall output as the summation of all incoming signals:

$O_{5, i}=\sum \widetilde{w}_{i} * f_{i}=\frac{\sum w_{i} * f_{i}}{\sum w_{i}}$

\subsection{Hamacher T-norm}

Hamacher T-norm as a kind of T-norms with parameter, satisfies boundary conditions ,commutativity, associativity and monotonicity. The parameter of Hamacher T-norm is also monotonous, and its expression is given below:

$T_{\lambda}(x, y)=\frac{x y}{\lambda+(1-\lambda)(x+y-x y)}$

where $\lambda>0$. Especially, when $\lambda=1$, Hamacher T-norm equals to algebraic product T-norm.

It is easy to recognise that algebraic product T-norm is an special Hamacher T-norm which has a constant parameter $\lambda$. However, employing a constant parameter $\lambda$ is not always appropriate. For any rule, there must be a corresponding parameter $\lambda$ suited for it. It is wise to use backpropagation algorithm to determine the corresponding $\lambda$.

\section{PROPOSED SYSTEM}

The output of layer-2 $O_{2,2(i-1)+j}$ refers to the result of intersection operation between $\mu_{A_{i}}(x)$ and $\mu_{B_{j}}(y)$, which means the membership degree that $\mathrm{x}^{1}$ belongs to $A_{i}$ and $\mathrm{x}^{2}$ belongs to $B_{j}$. It is common to use algebraic product T-norm $" * "$ to deal with the membership degree in intersection operation, but as is well-known that algebraic product T-norm is not proper in any situation. What (8) shows is that, algebraic product T-norm is a special Hamacher T-norm whose parameter is constant to 1 . So modifying the parameter to suit to the data pairs is a meaningful way to overcome the dilemma. It is not easy to determine the value of $\lambda$ that should be served in Hamacher T-norm to handle intersection operation. Iliadis et al. have tried to use other constant $\lambda$ to obtain better performance but not all always resulted in good situation [1]. It is a good solution to make ANFIS to adap- 




Fig. (2). The structure of ANFIS combined with subtract clustering.

tively select its own $\lambda$ for each rule. If ANFIS could select $\lambda$ for each rule respectively , according to the training data pairs, it is more likely to fit to the performance curve and close to the inherent law. Back-propagation Algorithm could be adopted in the process of determining the parameter of each rule, but this method needs to obtain $\frac{\partial T_{\lambda}(x, y)}{\partial x}$ and $\frac{\partial T_{\lambda}(x, y)}{\partial \lambda}$ which is the gradient of $T_{\lambda}(x, y)$.

\subsection{Multi-input Hamacher T-norm}

Calculating $\frac{\partial T_{\lambda}(x, y)}{\partial x}$ and $\frac{\partial T_{\lambda}(x, y)}{\partial \lambda}$ is easy, ANFIS may have more than two inputs and how to calculate their gradients is a real problem. More attention should be paid on how to calculate their gradient with more than 2 inputs. Now the definition of multi-input Hamacher T-norm is given below.

$T_{\lambda}\left(A_{n}\right)$ is multi-input Hamacher T-norm on $A_{n}$ which has $\mathrm{n}$ elements, where $A_{n}=\left\{a_{1}, a_{2}, \cdots, a_{n}\right\}$ and $\forall i \in N^{+}, 2 \leq n, 0 \leq a_{i} \leq 1 . T_{\lambda}\left(A_{n}\right)=T_{\lambda}\left(T_{\lambda}\left(A_{n-1}\right), a_{n}\right)$. Especially, when $\mathrm{n}=2, T_{\lambda}\left(A_{2}\right)=T_{\lambda}\left(a_{1}, a_{2}\right)$.

The definition given above is recursive definition, in other word the meaning of upper layer is corresponding to the lower one and the lowest is clarified. To express it clearly, a useful tool $\chi^{j}\left(A_{n}\right)$ has been used. The definition and features of it are given below: $\chi^{j}\left(A_{n}\right)=$ $\sum_{c_{1}, \cdots, c_{j} \in\{1, \cdots, n\}, c_{1} \neq \cdots \neq c_{j}} a_{c_{1}} a_{c_{2}} a_{c_{3}} \cdots a_{c_{j}}$, where $n \in N^{+}, \mathrm{n} \geq 2$ and $j \in N, j \leq n$. Especially, $\chi^{0}\left(A_{n}\right)=1$. For example, $\chi^{2}\left(A_{4}\right)=$ $a_{1} a_{2}+a_{1} a_{3}+a_{1} a_{4}+a_{2} a_{3}+a_{2} a_{4}+a_{3} a_{4}$.

Corollary 1 When $j \neq n$,

$\chi^{j}\left(A_{n}\right)=\chi^{j}\left(A_{n-1}\right)+a_{n} \chi^{j-1}\left(A_{n-1}\right)$

The proof is given in Appendix A.
Corollary 2 When $\mathrm{j}=\mathrm{n}$,

$\chi^{n}\left(A_{n}\right)=a_{n} \chi^{n-1}\left(A_{n-1}\right)$

The proof is given in Appendix A.

Corollary $3 \frac{\partial \chi^{j}\left(A_{n}\right)}{\partial a_{k}}=\chi^{j-1}\left(A_{n} \backslash a_{k}\right)$, where $\mathrm{j}, \mathrm{k} \in \mathrm{N}$ and $\mathrm{j}, \mathrm{k} \neq \mathrm{n} . A_{n} \backslash a_{k}=\left\{a_{1}, a_{2}, \cdots, a_{k-1}, a_{k+1}, \cdots, a_{n}\right\}$

The proof is given in Appendix A.

One evident feature of multi-input Hamacher T-norm is the monotonicity with respect to $\lambda$. After concise proof, the feature and the expression of $T_{\lambda}\left(A_{n}\right)$ are confirmed below:

Proposition $1 T_{\lambda}\left(A_{n}\right)$ is decreasing with respect to $\lambda$. Especially, when $\forall i \in[1, n+1] \quad a_{i} \neq 1$ and $a_{i} \neq 0, T_{\lambda}\left(A_{n}\right)$ is strictly decreasing with respect to $\lambda$.

The proof is given in Appendix B.

\section{Proposition 2}

$T_{\lambda}\left(A_{n}\right)=$

$\frac{\chi^{n}\left(A_{n}\right)}{\lambda^{n-1}+\sum_{j=1}^{n-1} \lambda^{n-j-1}(1-\lambda)^{j} \chi^{j}\left(A_{n}\right)-\sum_{i=1}^{n-1}(1-\lambda)^{i} \chi^{n}\left(A_{n}\right)}$

The proof is given in Appendix B

Proposition $3 \frac{\partial T_{\lambda}\left(A_{n}\right)}{\partial \lambda}=\frac{-\chi^{n}\left(A_{n}\right) R_{n}}{Q_{n}^{2}}$, where $Q_{n}=$ $\lambda^{n-1}+\sum_{i=1}^{n-1} \lambda^{n-i-1}(1-\lambda)^{i} \chi^{i}\left(A_{n}\right)-\sum_{i=1}^{n-1}(1-\lambda)^{i} \chi^{n}\left(A_{n}\right)$ and

$R_{n}=$

$(n-1) \lambda^{n-2}+\sum_{i=1}^{n-1} \lambda^{n-i-2}(1-\lambda)^{i-1}[(n-1)-(n-1) \lambda-i] \chi^{i}\left(A_{n}\right)+$ $\sum_{i=1}^{n-1} i(1-\lambda)^{i-1} \chi^{n}\left(A_{n}\right)$

The proof is given in Appendix B. 


\subsection{Hamacher T-norm and Subtract Clustering based ANFIS}

The proposed model differs from regular ANFIS in two points:

1. It makes Hamacher parameter variable and adaptive by adopting back-propagation algorithm, and needs to calculate the gradient with respect to each parameter and input.

2. It's combined with subtract clustering and employ it to determine the amount and value of each rule.

As is given above, the gradient of Hamacher T-norm's parameter and inputs have been achieved by Proposition 3 and Proposition 4. Different from the regular ANFIS, the output of layer-2 for a proposed model which has 3 inputs is given below:

$O_{2,3^{2}(i-1)+3(j-1)+k}=T_{\lambda}\left(A_{3}\right)$

where $A_{3}=\left\{\mu_{A_{i}}(x), \mu_{B_{j}}(y), \mu_{C_{k}}(z)\right\}$.

Each rule has same position in regular ANFIS, because their $\lambda$ have been uniformly set to 1 , which hammers the system to find the most significant rule adaptively. However, proposed model's each rule with different $\lambda$ in the end can lay the foundation for measuring the importance of itself. Both the IF part and the THEN part correlate to the $\lambda$ and the principle of updating $\lambda$ is to minimize the error, which guarantees that updated $\lambda$ is harmonious to the system. ${ }^{w_{i}}$ is the weight of $i$ th rule and decreasing with respect to $\lambda$ according to Proposition 1 and Equation(9). It means that the less $\lambda$ leads to bigger $w_{i}$, then bigger $\widetilde{w}_{i}$, so the $\mathbf{i}$ th rule plays a more important role in proposed model.

In addition, this model involves the field that the others have never touched upon. This field is attached to the improvement in fuzzy reasoning, and it could be combined with the improvement both in fuzzy reasoning and in other process, because it provides a new methodology for handling intersection operation.

With the variable and adaptive parameter, the prediction ability of proposed model may be improved; the parameter is modified according to the gradient and so as to fit to the inherent law. Empirical study will be given in next section, which proves that the proposed one overweighs the regular ANFIS.

\section{EXPERIMENT}

Famous Box-Jenkins dataset is the benchmark dataset to validate the performance of fitting method. The Box-Jenkins dataset represents the $\mathrm{CO}_{2}$ concentration as output, $\mathrm{y}(\mathrm{t})$, in terms of input gas flow rate, $u(t)$, form a combustion process of a methane air mixtrue.Lots of early work has been done on fitting Box-Jenkins dataset.Among them 7 input-type has been widely used:(A) u(k-4),y(k-1); (B) u(k-3), y(k-1); (C) $\mathrm{u}(\mathrm{k}-3), \mathrm{u}(\mathrm{k}-4), \mathrm{y}(\mathrm{k}-1)$; (D) $\mathrm{u}(\mathrm{k}), \mathrm{u}(\mathrm{k}-1), \mathrm{y}(\mathrm{k}-1), \mathrm{y}(\mathrm{k}-2) ;(\mathrm{E})$ $\mathrm{u}(\mathrm{k}-1), \mathrm{u}(\mathrm{k}-2), \mathrm{y}(\mathrm{k}-1), \mathrm{y}(\mathrm{k}-2) ;(\mathrm{F}) \mathrm{u}(\mathrm{k}), \mathrm{u}(\mathrm{k}-1), \mathrm{u}(\mathrm{k}-2), \mathrm{y}(\mathrm{k}-1)$, $\mathrm{y}(\mathrm{k}-2), \mathrm{y}(\mathrm{k}-3)$; (G) $\mathrm{u}(\mathrm{k}-1), \mathrm{u}(\mathrm{k}-2), \mathrm{u}(\mathrm{k}-3), \mathrm{y}(\mathrm{k}-1), \mathrm{y}(\mathrm{k}-2), \mathrm{y}(\mathrm{k}-$ $3)$.
Whole experiment was undertoken in the environment of Matlab 7.8.0. The results of proposed model and early work are listed in Table 1, where we can find that the proposed model has an outstanding performance.

\section{CONCLUSION}

Hamacher T-norm is one of the most influential T-norms. In this paper, we investigate the feasibility of applying ANFIS implemented with Hamacher T-norm. While employing benchmark Box-Jenkins dataset, the proposed methods have a more competitive performance in prediction accuracy compared to early work.

There are two main advantage of proposed model: on the one hand, it is the extention of ANFIS in fuzzy reasoning, and makes it possible to improve when be implemented with other improvement in fuzzification, defuzzification even training method and other optimal algorithm such as GA and PSO; on the other hand, it provides a very vital parameter $\lambda$ to infer the importance of each rule, but the normal form of inferring and measuring has not been proposed. The study of all above expectations is in progress.

Table 1. The results of each model.

\begin{tabular}{|c|c|c|}
\hline Model & Input-type & MSE \\
\hline \hline PMDE [4] & F & 0.1247 \\
\hline PMGA [4] & F & 0.3508 \\
\hline TS-GMDH1 [9] & G & 0.1299 \\
\hline TS-GMDH2 [9] & G & 0.2197 \\
\hline TS-GMDH3 [9] & G & 0.3310 \\
\hline Mejias [3] & B & 0.3129 \\
\hline Yue [8] & A & 0.1480 \\
\hline Yue [8] & B & 0.1240 \\
\hline Yue [8] & C & 0.1030 \\
\hline Yue [8] & F & 0.0460 \\
\hline Yue [8] & G & 0.0420 \\
\hline Proposed Model & F & 0.0325 \\
\hline Proposed Model & G & 0.0301 \\
\hline
\end{tabular}

\section{CONFLICT OF INTEREST}

The authors confirm that this article content has no conflict of interest.

\section{ACKNOWLEDGEMENTS}

The authors would like to thank for the support by innovation Project of Guangxi Graduate Education (YCSZ2014203), Project of Outstanding Young Teachers' Training in Higher Education Institutions of Guangxi and a grant of Philosophy and Social Science Fund of Guangxi (13BGL009). 


\section{APPENDIX A. PROOF OF COROLLARY}

$$
\begin{aligned}
& \text { Proof of Corollary } 1 \text { When } 0 \leq j<n, 1 \leq i \leq n \\
& \chi^{j}\left(A_{n}\right)=\sum_{c_{1}, \cdots, c_{j} \in\{1, \cdots, i-1, i+1, \cdots, n\}, c_{1} \neq \cdots \neq c_{j}}\left(a_{c_{1}} a_{c_{2}} a_{c_{3}} \cdots a_{c_{j}}\right) \\
& =\sum_{c_{1}, \cdots, c_{j} \in\left\{1, \cdots, i-1, i+1, \cdots, n, c_{1} \neq \cdots \neq c_{j}\right.}\left(a_{c_{1}} a_{c_{2}} a_{c_{3}} \cdots a_{c_{j}}\right) \\
& +a_{i} \sum_{c_{1}, \cdots, c_{j}-1 \in\left\{\{1, \cdots, i-1, i+1, \cdots, n\}, c_{1} \neq \cdots \neq c_{j-1}\right.}\left(a_{c_{1}} a_{c_{2}} a_{c_{3}} \cdots a_{c_{j-1}}\right) \\
& =\chi^{j}\left(A_{n} \backslash a_{i}\right)+a_{i} \chi^{j-1}\left(A_{n} \backslash a_{i}\right)
\end{aligned}
$$

Which completes the proof.

Proof of Corollary 2 When $j \neq n$,

$$
\begin{aligned}
& \chi^{n}\left(A_{n}\right)=a_{1} a_{2} a_{3} \cdots a_{n} \\
& =a_{n}\left(a_{1} a_{2} a_{3} \cdots a_{n}\right) \\
& =a_{n} \chi^{n}\left(A_{n}\right)
\end{aligned}
$$

Which completes the proof.

Proof of Corollary 3 When $0 \leq j<n, 1 \leq i \leq n$

$$
\begin{aligned}
& \frac{\partial \chi^{j}\left(A_{n}\right)}{\partial a_{i}}=\frac{\partial \chi^{j}\left(A_{n} \backslash a_{i}\right)}{\partial a_{i}}+\frac{\partial\left(a_{i} \chi^{j-1}\left(A_{n} \backslash a_{i}\right)\right)}{\partial a_{i}} \\
& =\chi^{j-1}\left(A_{n} \backslash a_{i}\right)
\end{aligned}
$$

Which completes the proof.

\section{APPENDIX B. PROOF OF PROPOSITION}

Proof of Proposition $1 \forall \lambda_{1}, \lambda_{2} \in[0,+\infty]$ and $\lambda_{1}<\lambda_{2}$, when $\mathrm{n}=1, \quad T_{\lambda_{1}}\left(A_{2}\right)=T_{\lambda_{1}}\left(a_{1}, a_{2}\right) \geq T_{\lambda_{2}}\left(a_{1}, a_{2}\right) \geq T_{\lambda_{2}}\left(A_{2}\right)$. Especially, when $a_{1}, a_{2} \neq 1$ and $a_{1}, a_{2} \neq 0, T_{\lambda}\left(A_{n}\right)$ is strictly decreasing with respect to $\lambda$. The proposition is confirmed.

Assume when $\mathrm{n}=\mathrm{t}-1$, the proposition is right too, then when $\mathrm{n}=\mathrm{t}, \quad T_{\lambda_{1}}\left(A_{t+1}\right)=T_{\lambda_{1}}\left(T_{\lambda_{1}}\left(A_{t}\right), a_{t+1}\right) \quad \geq T_{\lambda_{1}}\left(T_{\lambda_{2}}\left(A_{t}\right), a_{t+1}\right)$ $\geq T_{\lambda_{2}}\left(T_{\lambda_{2}}\left(A_{t}\right), a_{t+1}\right)=T_{\lambda_{2}}\left(A_{t+1}\right)$. Especially, when $\forall i \in[0, n+1]$ $a_{i} \neq 1$ and $a_{i} \neq 0, T_{\lambda}\left(A_{n}\right)$ is strictly decreasing with respect to $\lambda$.

Which completes the proof.

Proof of Proposition 2 The proof is given below:

Let $\mathrm{Q}_{n}=\lambda^{n-1}+\sum_{j=1}^{n-1} \lambda^{n-j-1}(1-\lambda)^{j} \chi^{j}\left(A_{n}\right)-\sum_{i=1}^{n-1}(1-\lambda)^{i} \chi^{n}\left(A_{n}\right)$, so $T_{\lambda}\left(A_{n}\right)=\frac{\chi^{n}\left(A_{n}\right)}{Q_{n}}$.

When $n=2$,

$$
\begin{aligned}
& T_{\lambda}\left(A_{2}\right)=\frac{a_{1} a_{2}}{\lambda+(1-\lambda)\left(a_{1}+a_{2}-a_{1} a_{2}\right)} \\
& =\frac{a_{1} a_{2}}{\lambda+(1-\lambda)\left(a_{1}+a_{2}\right)+(\lambda-1) a_{1} a_{2}}
\end{aligned}
$$

$$
\begin{aligned}
& =\frac{\chi^{2}\left(A_{2}\right)}{\lambda^{2-1}+\sum_{j=1}^{2-1} \lambda^{1-j}(1-\lambda)^{j} \chi^{j}\left(A_{2}\right)-\sum_{i=1}^{2-1}(\lambda-1)^{i} \chi^{2}\left(A_{2}\right)} \\
& =\frac{\chi^{2}\left(A_{2}\right)}{Q_{2}}
\end{aligned}
$$

The proposition is right.

Assume when $\mathrm{n}=\mathrm{t}$, proposition is right too. So, $T_{\lambda}\left(A_{t}\right)=\frac{\chi^{t}\left(A_{t}\right)}{Q_{t}}$.

$$
\begin{aligned}
& T_{\lambda}\left(A_{t+1}\right)=\frac{\frac{\chi^{t}\left(A_{t}\right)}{Q_{t}} a_{t+1}}{\lambda+(1-\lambda)\left(\frac{\chi^{t}\left(A_{t}\right)}{Q_{t}}+a_{t+1}\right)-(1-\lambda) a_{t+1} \frac{\chi^{t}\left(A_{t}\right)}{Q_{t}}} \\
& =\frac{\chi^{t+1}\left(A_{t+1}\right)}{\lambda Q_{t}+(1-\lambda) \chi^{t}\left(A_{t}\right)-(1-\lambda) \chi^{t+1}\left(A_{t+1}\right)+(1-\lambda) a_{t+1} Q_{t}} \\
& \lambda Q_{t}=\lambda \sum_{j=0}^{t-1} \lambda^{t-j-1}(1-\lambda)^{j} \chi^{j}\left(A_{t}\right)+\lambda \frac{(1-\lambda)^{t}-(1-\lambda)}{\lambda} \chi^{t}\left(A_{t}\right) \\
& =\sum_{j=0}^{t-1} \lambda^{t-j}(1-\lambda)^{j} \chi^{j}\left(A_{t}\right)+\left[(1-\lambda)^{t}-(1-\lambda)\right] \chi^{t}\left(A_{t}\right) \\
& (1-\lambda) a_{t+1} Q_{t} \\
& =\sum_{j=0}^{t-1} \lambda^{t-j-1}(1-\lambda)^{j+1} a_{t+1} \chi^{j+1-1}\left(A_{t}\right)+\frac{\left[(1-\lambda)^{t+1}-(1-\lambda)^{2}\right]}{\lambda} a_{t+1} \chi^{t}\left(A_{t}\right) \\
& =\sum_{j=1}^{t} \lambda^{t-j}(1-\lambda)^{j} a_{t+1} \chi^{j-1}\left(A_{t}\right)+\frac{\left[(1-\lambda)^{t+1}-(1-\lambda)^{2}\right]}{\lambda} \chi^{t+1}\left(A_{t+1}\right) \\
& \lambda Q_{t}+(1-\lambda) \chi^{t}\left(A_{t}\right)-(1-\lambda) \chi^{t+1}\left(A_{t+1}\right)+(1-\lambda) a_{t+1} Q_{t} \\
& =\lambda^{t}+\sum_{j=1}^{t-1} \lambda^{t-j}(1-\lambda)^{j} \chi^{j}\left(A_{t}\right)+\sum_{j=1}^{t} \lambda^{t-j}(1-\lambda)^{j} a_{t+1} \chi^{j-1}\left(A_{t}\right) \\
& +\left[(1-\lambda)^{t}-(1-\lambda)\right] \chi^{t}\left(A_{t}\right)+\frac{\left[(1-\lambda)^{t+1}-(1-\lambda)^{2}\right]}{\lambda} a_{t+1} \chi^{t+1}\left(A_{t+1}\right) \\
& \left.+(1-\lambda) \chi^{t}\left(A_{t}\right)-(1-\lambda) \chi^{t+1}\left(A_{t+1}\right)\right) \\
& =\lambda^{t}+\sum_{j=1}^{t-1} \lambda^{t-j}(1-\lambda)^{j}\left(\chi^{j}\left(A_{t}\right)+a_{n+1} \chi^{j-1}\left(A_{t}\right)\right. \\
& +(1-\lambda)^{t} f\left(a_{t+1}\right) \chi_{t-1, f(\cdot)}^{t-1}\left(A_{t}\right)+(1-\lambda)^{t} \chi^{t}\left(A_{t}\right)-(1-\lambda) \chi^{t+1}\left(A_{t+1}\right) \\
& +\frac{\left[(1-\lambda)^{t+1}-(1-\lambda)^{2}\right]}{\lambda} \chi^{t+1}\left(A_{t+1}\right) \\
& =\lambda^{t}+\sum_{j=1}^{t-1} \lambda^{t-j}(1-\lambda)^{j} \chi^{j}\left(A_{t+1}\right) \\
& +(1-\lambda)^{t}\left(\chi^{t}\left(A_{t+1}\right)-\chi^{t}\left(A_{t}\right)+(1-\lambda)^{t} \chi^{t}\left(A_{t}\right)\right. \\
& +\frac{(1-\lambda)^{t+1}-(1-\lambda)^{2}-\lambda+\lambda^{2}}{\lambda} \chi^{t+1}\left(A_{t+1}\right) \\
& =\lambda^{t}+\sum_{j=1}^{t-1} \lambda^{t-j}(1-\lambda)^{j} \chi^{j}\left(A_{t+1}\right)+(1-\lambda)^{t} \chi^{t}\left(A_{t+1}\right)+\frac{(1-\lambda)^{t+1}-(1-\lambda)}{\lambda} \chi^{t+1}\left(A_{t+1}\right) \\
& =\lambda^{t}+\sum_{j=1}^{t} \lambda^{t-j}(1-\lambda)^{j} \chi^{j}\left(A_{t+1}\right)+\frac{(1-\lambda)^{t+1}-(1-\lambda)}{\lambda} \chi^{t+1}\left(A_{t+1}\right) \\
& =\lambda^{t}+\sum_{j=1}^{t} \lambda^{t-j}(1-\lambda)^{j} \chi^{j}\left(A_{t+1}\right)-\sum_{i=1}^{t}(1-\lambda)^{i} \chi^{t+1}\left(A_{t+1}\right)
\end{aligned}
$$


$=Q_{t+1}$

$$
\text { So, } T_{\lambda}\left(A_{t+1}\right)=\frac{\chi^{t+1}}{Q_{t+1}} \text {. }
$$

In
$T_{\lambda}\left(A_{n}\right)=\frac{\text { conclusion, }}{\chi^{n}\left(A_{n}\right)} \quad n \in N^{+}$
$\lambda^{n-1}+\sum_{j=1}^{n-1} \lambda^{n-j-1}(1-\lambda)^{j} \chi^{j}\left(A_{n}\right)-\sum_{i=1}^{n-1}(1-\lambda)^{i} \chi^{n}\left(A_{n}\right)$

Which completes the proof.

\section{Proof of Proposition 3}

$$
\frac{\partial Q_{n}}{\partial \lambda}
$$$$
={ }_{(n-1)} \lambda^{n-2}+\sum_{i=1}^{n-1}(n-i-1) \lambda^{n-i-2}(1-\lambda)^{i} \chi^{i}\left(A_{n}\right)-\lambda^{n-i-1}(1-\lambda)^{i-1} \chi^{i} A_{n}
$$$$
+\sum_{i=1}^{n-1} i(1-\lambda)^{i-1} \chi^{n}\left(A_{n}\right)
$$

$$
=
$$$$
(n-1) \lambda^{n-2}+\sum_{i=1}^{n-1} \lambda^{n-i-2}(1-\lambda)^{i-1}((n-1)-(n-1) \lambda-i) \chi^{i}\left(A_{n}\right)+\sum_{i=1}^{n-1} i(1-\lambda)^{i-1} \chi^{n}\left(A_{n}\right)
$$$$
=R_{n}
$$

$$
\frac{\partial T_{n}\left(A_{n}\right)}{\partial \lambda}=\frac{\partial \frac{\chi^{n}\left(A_{n}\right)}{Q_{n}}}{\partial \lambda}=\frac{-\chi^{n}\left(A_{n}\right) R_{n}}{Q_{n}^{2}},
$$

Which completes the proof.

\section{REFERENCES}

[1] L.S. Iliadis, S. Spartalis and S. Tachos,2008. Application of fuzzy T-norms towards a new Artificial Neural Networks evaluation framework: A case from wood industry, Information Sciences, 178, 3828-3839.

[2] J.S. R, 1993. ANFIS: Adaptive-network- based fuzzy inference systems, IEEE Trans. Syst., Man, Cybern., 23 (3), 665-685.

[3] Mejas, A., Romero, S. and Moreno, F.J., 2008. A Systematic Methodology to Obtain a Fuzzy Model Using an Adaptive Neuro Fuzzy Inference System. Application for Generating a Model for GasFurnace Problem, Distributed Computing and Artificial Intelligence, 452-460.DOI: 10.1007/978-3-540-85863-8_53.

[4] Ozer S. and Zorlu H., 2011. Identification of bilinear systems using differential evolu-tion algorithm, Sadhana-academy Pro-ceedings in Engineering Sciences, 36(3), 281-292. DOI: 10.1007/s12046-011$0022-8$.

[5] M. Sugeno, G. T. Kang, 1988. Structure identification of fuzzy model, Fuzzy Sets Syst., 28(1), 15-33.

[6] M. Sugeno, K. Tanaka, 1991. Successive identification of a fuzzy model and its applications to prediction of a complex system, Fuzzy Sets Syst., 42(3), 315-334.

[7] T. Takagi, I. Hayashi, 1991. NN-driven fuzzy reasoning, Int. J. Approx. Reason., 5(3), 191-212.

[8] Yue, J.H., Liu, J.Z., Liu X.J. and Tan, W., 2006. Identification of Nonlinear System Based on ANFIS with Subtractive Clustering,Proceedings of the 6th World Congress on Intelligent Control and Automation, 1, 1852-1856. DOI: 10.1109/WCICA.2006.1712675

[9] Zhu, B., He C.-Z., Liatsisb P. and Li X.Y., 2012. A GMDH-based fuzzy modeling approach for constructing TS-model, Fuzzy Sets and Systems, 189, 19-29. DOI: 10.1016/j.fss.2011.08.004

Received: September 16, 2014

Revised: December 23, 2014

Accepted: December 31, 2014

(C) Zhang and Liao; Licensee Bentham Open.

This is an open access article licensed under the terms of the Creative Commons Attribution Non-Commercial License (http://creativecommons.org/licenses/by-nc/3.0/) which permits unrestricted, non-commercial use, distribution and reproduction in any medium, provided the work is properly cited. 